\title{
Fractional amplitude of low-frequency fluctuation in patients with neovascular glaucoma: a resting-state functional magnetic resonance imaging study
}

\author{
Yu-Qing Zhang ${ }^{\sharp}$, Meng-Ying Peng ${ }^{\#}$, Shi-Nan Wü ${ }^{\#}$ Chen-Yu Yu, Si-Yi Chen, Si-Wen Tan, Yi Shao^, \\ Qiong Zhou^
}

Department of Ophthalmology, The First Affiliated Hospital of Nanchang University, Jiangxi Province Ocular Disease Clinical Research Center, Nanchang, China

\#These authors contributed equally to this work.

Correspondence to: Yi Shao; Qiong Zhou. Department of Ophthalmology, The First Affiliated Hospital of Nanchang University, No 17, YongWaiZheng Street, DongHu District, Nanchang 330006, China. Email: freebee99@163.com; qiongz-ms@126.com.

Background: Neovascular glaucoma (NVG) is a secondary refractory disease with a poor prognosis, and there are few advanced studies on its pathogenesis and treatment. In this research, the fractional amplitude of low-frequency fluctuation (fALFF) technology was used in resting-state functional magnetic resonance imaging (rsfMRI) to investigate intrinsic neuron activity in the patient's brain with NVG.

Methods: Sixteen patients with NVG (eight males and eight females) and 16 healthy controls (HCs) of similar age and sex were included. All patients and controls received rsfMRI scans, and the differences between the two groups in fALFF values were compared by independent sample t-test. Receiver operating characteristic (ROC) curves were used to compare fALFF values in the brain regions of NVG patients and HCs and assess accuracy. Finally, Pearson linear correlation analysis assessed the correlation between fALFF signals in brain regions and the clinical evaluation indicators of patients with NVG.

Results: In patients with NVG, fALFF signal values in the right Rolandic operculum, left anterior cingulate and paracingulate gyri, and right caudate were significantly decreased. In contrast, fALFF signal values in the left precuneus were significantly higher than those recorded in the HCs. Analysis of the ROC curve for each brain region showed that the area under the ROC curve of NVG patients was large (close to 1), and the accuracy was good. In the NVG group, the hospital anxiety and depression scale $(\mathrm{r}=-0.952, \mathrm{P}<0.001)$ and left best-corrected visual acuity $(\mathrm{r}=-0.802, \mathrm{P}<0.001)$ had a negative linear correlation with the fALFF signal value of the right Rolandic operculum. The hospital anxiety and depression scale had a negative linear correlation with the fALFF signal value of the right caudate $(\mathrm{r}=-0.948, \mathrm{P}<0.001)$.

Conclusions: NVG patients showed dysfunction in several brain regions. These findings may assist in revealing the underlying neural mechanism of brain activity associated with NVG.

Keywords: Fractional amplitude of low-frequency fluctuation (fALFF); inner brain activity; neurodegenerative disease; neovascular glaucoma (NVG); resting-state functional magnetic resonance imaging (rsfMRI)

Submitted Jul 12, 2020. Accepted for publication Dec 31, 2020.

doi: 10.21037/qims-20-855

View this article at: http://dx.doi.org/10.21037/qims-20-855

^ ORCID: Yu-Qing Zhang, 0000-0001-8096-0789; Yi Shao, 0000-0003-1571-2433; Qiong Zhou, 0000-0002-1810-3775. 


\section{Introduction}

Neovascular glaucoma (NVG) is a secondary refractory disease, accounting for $0.7-5.1 \%$ of glaucoma cases in Asians $(1,2)$. It is typically characterized by iris neovascularization, eventual angle closure in the anterior chamber angle, and markedly increased intraocular pressure (IOP) (3). New blood vessels initially appear at the edge of the pupil and subsequently appear on the surface of the iris and anterior chamber angle. Coats first reported the growth of new blood vessels on the surface of the iris in 1906. Weiss et al. (4) coined the term "neovascularization glaucoma" in 1963 based on the presence of new blood vessels and associated connective tissue related to IOP. The three most common causes of NVG are diabetic retinopathy, ischemic central retinal vein occlusion, and ocular ischemic syndrome, accounting for $33 \%, 33 \%$, and $13 \%$ of cases, respectively. Other causes include chronic retinal detachment, intraocular neoplasms, severe intraocular inflammation, and vascular diseases (e.g., central retinal artery occlusion and branch retinal vein occlusion). These conditions are among the numerous disorders that cause anterior-segment neovascularization (5). The control of NVG involves two key aspects: (I) treatment of potential diseases that may lead to the early development of neovascularization, and (II) management of the increase in IOP (6). A pathological increase in IOP is the main risk factor for glaucoma, which can cause optic neuropathy and ultimately lead to ganglion cell death (7). Using functional magnetic resonance imaging ( $\mathrm{fMRI}$ ), neuroimaging studies have revealed damage in the anterior visual pathway and cerebral cortex. A common feature of most neurodegenerative diseases is the presence of protein aggregates in the central nervous system, and deposits of misfolded proteins have been found in the visual cortex and optic nerve of glaucoma patients. This shows that glaucoma is a disease that damages the visual pathway and neurodegenerative disease that influences numerous brain regions (8-11). Unfortunately, the mechanism of brain damage and optic neuropathy related to glaucoma has not been thoroughly studied. Most studies have focused on primary open-angle glaucoma and primary angleclosure glaucoma, with little advanced research examining NVG (12). Our team has conducted studies on brain activity changes in patients with normal-tension glaucoma and primary angle-closure glaucoma, and abnormal spontaneous activity has been detected in some brain regions $(13,14)$. In this study, we used neuroimaging to study NVG's brain processes and sought to uncover the neural mechanisms underlying brain activity in patients with NVG.

Resting-state fMRI (rsfMRI) is a promising method to research brain activity in clinical patients (15-18). It has become a beneficial tool for investigating functional connections in the human brain (19) and can draw circuits that constitute the physiological basis of information processing and psychological representation (20). As it is both convenient and minimally invasive, rsfMRI can skillfully recognize the functional areas in different patient groups, such as children, unconscious patients, and those with low intelligence quality (IQ) metric (21). By recognizing changes in magnetic resonance signals produced by alterations in blood oxygen levels, it detects spontaneous neuron activity in brain tissue. When brain neurons are active, an increase in different proportions of regional brain tissue blood flow and oxygen consumption is triggered, and the ratio of oxyhemoglobin to deoxyhemoglobin changes. This leads to changes in the local magnetic field's properties, resulting in enhanced magnetic resonance signals or weaken. According to the different properties of the two hemoglobin magnetic fields, MRI imaging methods can determine changes in local cerebral blood flow, thereby indirectly measuring the functional activities of the nervous system. Owing to its advantages of precise localization and combining functional and structural imaging without exposure to radioactive tracers, rsfMRI can provide new insight into the pathophysiology of glaucoma (22), and its use in the study of various neurodegenerative diseases and exploration of the mechanism of glaucoma is well established (23-26).

An important method for analyzing rsfMRI is to record spontaneous low-frequency fluctuations (LFF) in the brain. The amplitude of low-frequency fluctuations (ALFF) and fractional amplitude of low-frequency fluctuation (fALFF) are two indices thought to show the intensity of intrinsic spontaneous brain activity (24). As a normalized ALFF index, fALFF can specifically measure LFF in a specific frequency range (27) and detect spontaneous neural activity by measuring the low-frequency oscillation amplitude of brain blood flow $(0.01-0.08 \mathrm{~Hz})$. Zou et al. (27) showed that fALFF could effectively inhibit nonspecific signal components in the cistern areas, which means the sensitivity and specificity of spontaneous brain activity in the detection area can be significantly improved. In this study, we used rsfMRI to analyze fALFF in response to spontaneous brain activity in patients with NVG and healthy controls (HCs) and compare the values obtained in these two groups. This 
investigation may offer a deeper understanding of NVG and provide insight into its pathogenesis and treatment.

\section{Methods}

\section{Subjects}

This study included a group of 16 patients with NVG (eight males and eight females) and 16 sex-matched health controls (HCs). There was no significant difference in patients' age or sex in each group $(\mathrm{P}>0.05)$. The study was approved by the Human Research Ethics Committee of the First Affiliated Hospital of Nanchang University (Nanchang, China). We fully described the study's purpose to the subjects, and all subjects provided informed consent before their participation. The local Human Research Subcommittee approved all experimental protocols following the tenets of the Declaration of Helsinki.

The First Affiliated Hospital of Nanchang University recruited the patients with NVG, while HCs were recruited from the local area. The inclusion criteria for patients with NVG were as follows: (I) clinical diagnosis of NVG (diagnostic criteria for NVG: typical iris neovascularization and pupillary pigmentary valgus, neovascularization of the angular trabecular meshwork, peripheral anterior adhesion, IOP elevation, decreased vision, previous primary disease, characteristic glaucoma of visual field defect and cup); (II) persistent IOP $>21 \mathrm{mmHg}$ despite the use of IOP-lowering drugs; (III) absence of other eye diseases; (IV) absence of mental illness; and (V) right-handedness. The exclusion criteria were: (I) history of eye surgery; (II) neurological disease, hypertension, or diabetes; (III) inability to participate in MRI scans for subjective or objective reasons; and (IV) use of alcohol or psychotropic substances in the previous 3 months. Patients with hypertension were excluded because previous studies have found that hypertension causes gray matter abnormalities and other brain structural changes, affecting the results of this study. While diabetic retinopathy is the most common cause of NVG, long-term high blood sugar will also cause microvascular disease and neuropathy and synaptic degeneration in peripheral nerves, which could also affect our research results. On this basis, patients with diabetes were also excluded. The selection criteria for HCs were: (I) lack of history of eye diseases; (II) absence of abnormalities in the visual pathway and brain parenchyma as depicted on head MRI; (III) no contraindications to MRI; and (IV) normal neurology and mental evaluation (Figure 1).

\section{MRI examination}

A Siemens Trio 3.0T MRI scanner (Erlangen, Germany) with an 8-channel phased-array head coil was used for MRI scans. During the rsfMRI scan, subjects closed their eyes and maintained them in the center position while their head was fixed with a belt and foam pad to ensure they remained motionless. The lights in the examination room were left on during the examination. Functional data was acquired using an echo planar imaging sequence as follows: repeat time $=2,000 \mathrm{~ms}$; echo time $=30 \mathrm{~ms}$; flip angle $=90^{\circ}$; slice thickness $=3 \mathrm{~mm}$ with a $1-\mathrm{mm}$ gap; field of view $=240 \mathrm{~mm} \times 240 \mathrm{~mm}$. Each brain volume comprised 30 axial slices, and 240 functional images were finally captured. The entire process was completed in $8 \mathrm{~min}$.

\section{Data processing}

Functional data were analyzed through the MRIcro software (http://www.mricro.com), and SPM8 (http://www.fil.ion. ucl.ac.uk/spm) was used for fMRI image preprocessing. The first two volumes of the functional image were discarded to achieve magnetization balance, and the remaining 230 volumes were manipulated through a slice-timing correction procedure and realigned to the first volume to correct for head movements. A standard echo planar imaging templates was used for spatial standardization to meet the standard of Montreal Neurological Institute. (resampling voxel size $=3 \mathrm{~mm} \times 3 \mathrm{~mm} \times 3 \mathrm{~mm}$ ). Subsequently, a temporal bandpass filter $(0.01-0.08 \mathrm{~Hz})$ was applied to reduce the effects of low-frequency drift and high-frequency noise signals. Finally, the image was smoothed with $6 \mathrm{~mm}$ full-width at half-maximum Gaussian kernel to reduce spatial noise.

\section{fALFF analysis}

We used REST software (http://www.resting-fmri. Sourceforge.net) to perform fALFF measurements using the ratio of each frequency in the low-frequency range $(0.01-0.08 \mathrm{~Hz})$ to the power in the entire frequency range $(0-0.25 \mathrm{~Hz})$.

\section{Statistical analysis}

Statistical analysis was performed using SPSS version 19.0 software (IBM Corp., Armonk, NY, USA), and independent sample $t$-test and chi-square tests were used to compare demographic and clinical indicators between 

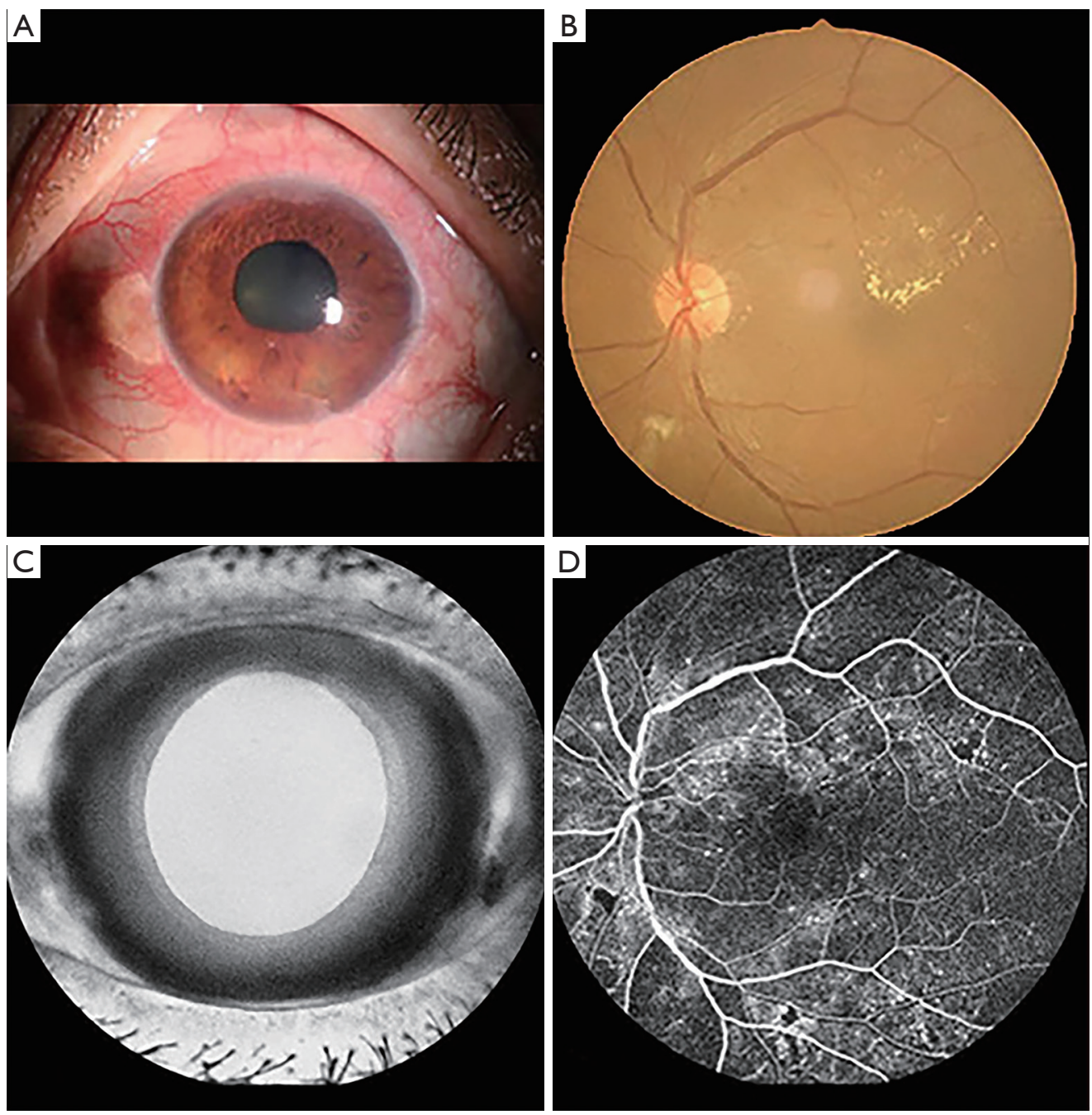

Figure 1 Eye examination results of NVG patients. Eye examination results for NVG patients are as follows: (A) results of anterior segment photography in NVG patients; (B) fundus photographic results; (C) results of iris fluorescein angiography in NVG patients; (D) FFA results of NVG patients. NVG, neovascular glaucoma; FFA, fundus fluorescein angiography.

the two groups. Differences in fALFF values between NVG patients and HCs were compared using a two-sample $t$-test in REST software, and $\mathrm{P}$ values $<0.05$ indicated statistical significance. The receiver operating characteristic (ROC) curve was used as a dichotomous variable model that detected whether NVG was present utilizing elevated fALFF values in different brain regions, as we hypothesized that fALFF values in different brain regions could be a potentially useful diagnostic marker for distinguishing between the NVG and HCs groups. The ROC curve was drawn to compare the fALFF values of the two brain regions. The correlation between the fALFF signal in the brain-related areas and the clinical evaluation indicators of patients with NVG was evaluated using Pearson linear correlation analysis. The area under the curve (AUC) of different brain regions represented the change of fALFF values in this brain region, which could then be used as the true positive rate for NVG and HC's differential diagnosis 1-AUC represented the false positive rate.

\section{Results}

\section{Demographics and clinical indicators}

There was no significant difference in sex (chi-square test, 
Table 1 Conditions of participants included in the study

\begin{tabular}{|c|c|c|c|c|}
\hline Condition & NVGs & $\mathrm{HCs}$ & $t$ & $P$ value \\
\hline Age (years) & $54.87 \pm 5.34$ & $53.14 \pm 5.97$ & 0.167 & 0.947 \\
\hline Weight (kg) & $61.24 \pm 7.65$ & $63.56 \pm 6.72$ & 0.158 & 0.973 \\
\hline Handedness & $16 \mathrm{R}$ & $16 \mathrm{R}$ & N/A & $>0.99$ \\
\hline BCVA-L & $0.31 \pm 0.15$ & $0.96 \pm 0.25$ & -5.653 & 0.021 \\
\hline BCVA-R & $0.24 \pm 0.11$ & $1.02 \pm 0.25$ & -5.653 & 0.017 \\
\hline IOP-L & $29.52 \pm 10.19$ & $15.92 \pm 4.08$ & 13.214 & 0.009 \\
\hline IOP-R & $27.21 \pm 11.27$ & $16.61 \pm 4.22$ & 11.669 & 0.011 \\
\hline
\end{tabular}

Independent sample $t$-test between NVG patients and HCs $(\mathrm{P}<0.05$ represented statistically significant differences). BCVA, best corrected visual acuity; HC, healthy control; L, left; R, right; N/A, not applicable; NVG, neovascular glaucoma; IOP, intraocular pressure.

$\mathrm{P}>0.99$ ), age (independent sample $t$-tests, $\mathrm{P}=0.947$ ), and weight (independent sample $t$-tests, $\mathrm{P}=0.973$ ) between the HCs and patients with NVG. The best-corrected visual acuity (BCVA) values for the left and right eyes of the NVG group were $0.31 \pm 0.15$ and $0.24 \pm 0.11$, respectively; and the IOP values for the left and right eyes were $29.52 \pm 10.19$ and $27.21 \pm 11.27$, respectively (Table 1).

\section{Differences in $f A L F F$}

Patients with NVG showed markedly higher fALFF values in the left precuneus (LP) than that seen in HCs. In contrast, fALFF values in the right Rolandic operculum (RRO), left anterior cingulate and paracingulate gyri (LCA), and right caudate (RC) decreased significantly in patients with NVG (Figure 2, Tables 2,3).

\section{ROC curve}

The ROC curve analyzed the average fALFF values of changes in brain areas. It was assumed that differences in fALFF values might be a potentially useful diagnostic marker to distinguish the NVG group from the HC group. The greater the AUC, the closer the curve is to the upper left corner, and the higher the diagnostic accuracy. Accuracy was considered low or high if the AUC was $0.5-0.7$ or 0.7-0.9, respectively, and the AUC of the following fALFF values in different brain regions was determined: LP $(0.967$, $\mathrm{P}<0.001)$, RRO (0.962, $\mathrm{P}<0.001)$, LCA (0.981, $\mathrm{P}<0.001)$, $\mathrm{RC}(0.924, \mathrm{P}<0.001)$ (Figure 3).

\section{Correlation analysis}

In the NVG group, the hospital anxiety and depression scale (HADS), and left BCVA had a negative linear correlation with the fALFF signal value of RRO. Moreover, HADS had a negative linear correlation with RC's fALFF signal value (Figure 4).

\section{Discussion}

To the best of our knowledge, fALFF is one of the most effective ways to measure spontaneous neuronal activity changes and has been successfully applied to the study of patients with neurodegenerative diseases. In addition to being a retinal disease, glaucoma is also a neurodegenerative disease that affects numerous brain regions, and fALLF has great potential for elucidating the neural mechanism of NVG (Table 4) (23-26). While rsfMRI has been used to study glaucoma in the retina and optic nerve in detail, the brain's activity as a whole has rarely been studied. Crish et al. (28) reported that the early disruption of axonal transport in glaucoma is initiated in the brain, and lesions progress from the distal to the proximal (eye). In recent years, an increasing number of studies have utilized rsfMRI to investigate patients' intrinsic functional activities with glaucoma and provide meaningful insights into its neural mechanisms. In this study, we confirmed that the brain's internal activities changed in the NVG group compared with the HC group. The fALFF values of RRO, LCA, and RC in patients with NVG were significantly reduced, 

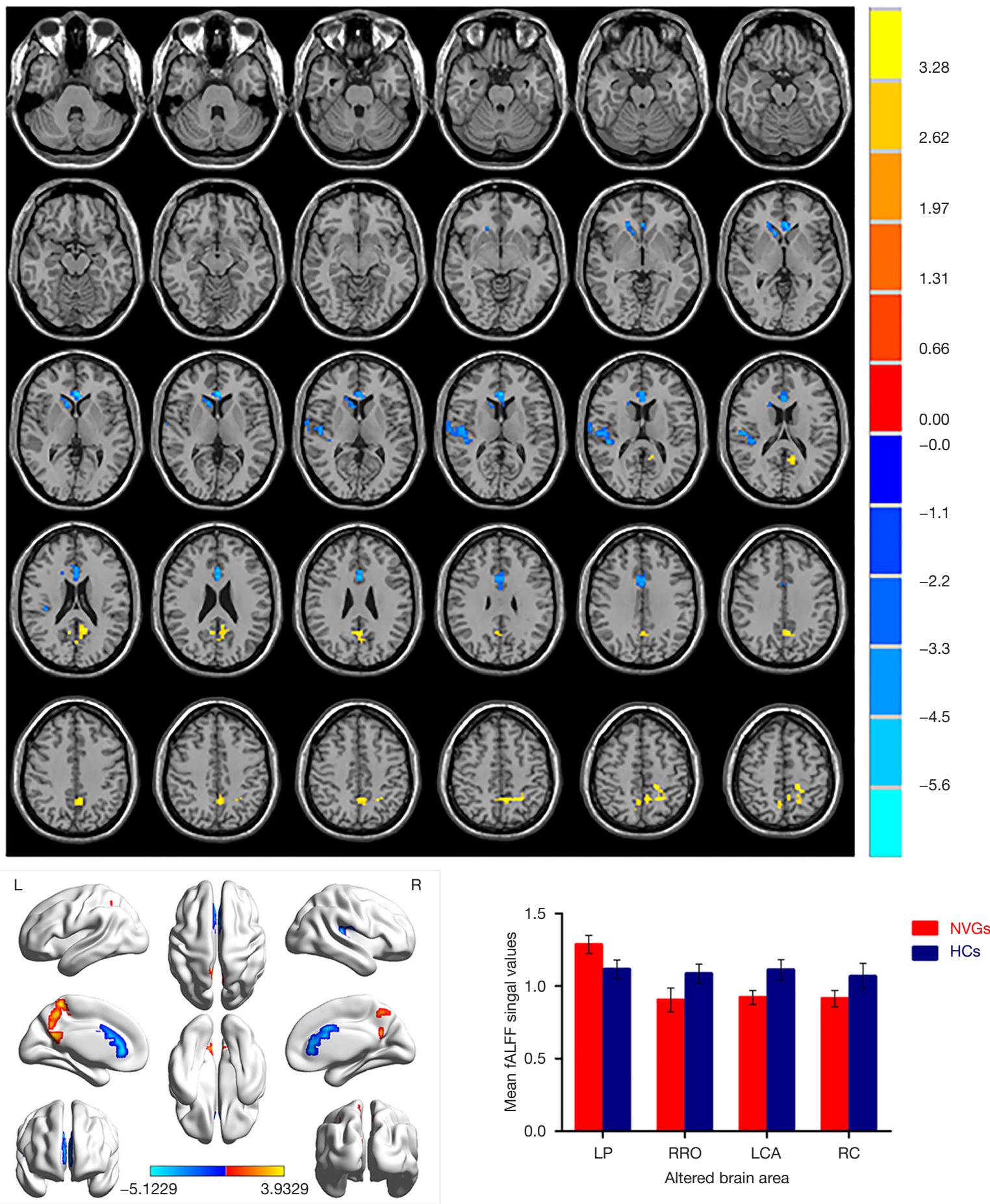

Figure 2 Spontaneous brain activity in patients with NVG versus HCs. MRI cross-sectional views and 3D images of the brain showed obvious differences in brain activity, with yellow areas representing higher fALFF values and blue areas representing lower fALFF values. The histogram shows the mean \pm standard deviation of the fALFF values for different brain regions between the two groups, with the red representing the NVG group and the dark blue representing the HC group. It shows that fALFF values in RRO, LCA, and RC decrease significantly in NVG patients, and fALFF values in LP increase significantly in NVG patients. fALFF, fractional amplitude of low-frequency fluctuation; NVG, neovascular glaucoma; HCs, healthy controls; RRO, right Rolandic operculum; RC, right caudate; LP, left precuneus; LCA, left anterior cingulate and paracingulate gyri; MRI, magnetic resonance imaging. 
Table 2 Brain regions where fALFF values differ significantly between NVG patients and HCs

\begin{tabular}{|c|c|c|c|c|c|c|c|}
\hline Brain areas & \multicolumn{3}{|c|}{ MNI coordinates } & BA & Number of voxels & $t$-value & ROI order \\
\hline \multicolumn{8}{|l|}{$\mathrm{NVG}>\mathrm{HC}$} \\
\hline LP & -18 & -36 & 54 & - & 248 & 3.9329 & 4 \\
\hline \multicolumn{8}{|l|}{$\mathrm{NVG}<\mathrm{HC}$} \\
\hline LCA & -3 & 33 & 3 & 24 & 200 & -5.1229 & 2 \\
\hline $\mathrm{RC}$ & 9 & 18 & 9 & 25 & 62 & -4.1344 & 1 \\
\hline
\end{tabular}

The statistical threshold was set at voxel with $P<0.01$. fALFF, fractional amplitude of low-frequency fluctuation; NVG, neovascular glaucoma; HC, healthy control; P, left precuneus; RRO, right Rolandic operculum; LCA, left anterior cingulum and paracingulate gyri; RC, right caudate; $\mathrm{ROI}$, region of interest.

Table 3 Independent sample $t$-tests between mean fALFF values between NVG patients and HCs related brain regions

\begin{tabular}{lcccc}
\hline Region & $\mathrm{HC}$ & $\mathrm{NVG}$ & $t$-value & $\mathrm{P}$ value \\
\hline LP & $1.115 \pm 0.066$ & $1.287 \pm 0.062$ & -7.566 & $\mathrm{P}<0.001$ \\
RRO & $1.085 \pm 0.065$ & $0.905 \pm 0.082$ & 6.901 & $\mathrm{P}<0.001$ \\
LCA & $1.112 \pm 0.071$ & $0.922 \pm 0.048$ & 8.816 & $\mathrm{P}<0.001$ \\
RC & $1.067 \pm 0.089$ & $0.914 \pm 0.056$ & 5.835 & $\mathrm{P}<0.001$ \\
\hline
\end{tabular}

Independent $t$-tests were compared between the two groups $(\mathrm{P}<0.05$ represents a statistically significant difference). fALFF, fractional amplitude of low-frequency fluctuation; NVGs, neovascular glaucoma subjects; HCs, healthy control subjects; LP, left precuneus; RRO, right Rolandic operculum; LCA, left anterior cingulate and paracingulate gyri; $\mathrm{RC}$, right caudate.

whereas those of the LP was significantly increased (Figure 5).

The precuneus is located in the anterior precuneus (upper occipital lobe) and connected to the cuneiform muscle and primary visual cortex's visual region. The precuneus is divided into the $7 \mathrm{M}$ and precuneus visual $(\mathrm{PCV})$ areas, and the $\mathrm{PCV}$ area is found in the anterior precuneus, posterior to the marginal ramus of the cingulate sulcus (29). Studies using fMRI have shown that the PCV area is involved in the process of recognizing emotional faces and working memory images of the face, body, and location (30-32). The $7 \mathrm{M}$ area is part of the precuneus involved in listening to stories over answering arithmetic problems; paying attention to social interaction objects in the geometry of random movement; recognizing the expression of these neutral objects; comparing the feature size of objects and match object according to language classification. Its other functions are the same as those performed by the PCV area (30-32). The precuneus is also essential in the function of visual space, which is essential for the integration of brain, body, and environment and the management of internal cognitive models (33-35). We found that patients with NVG showed they increased fALFF values of the LP when compared with HCs. We hypothesized that the anterior wedge's high activity might reflect obstacles in the visual space perception of patients with NVG and initiated neural compensation to maintain damaged neural reserve causing compensation for vision loss and visual field defects. Visual impairment plays an important role in the effect of the compensation mechanism.

The cingulate gyrus is a crescent-shaped brain gyrus located on the inner side of the brain. It is the main part of the limbic system and involves many functions, such as memory, cognitive function, and attention. Previous studies have shown that the anterior cingulate cortex plays a role in cognitive-related visual functions, in which the dorsal anterior cingulate cortex is involved in the true classification and recognition of visual objects (36-38). Our findings showed that the fALFF value of LCA in patients with NVG was decreased. This difference between HCs and NVGs may imply that the anterior cingulate and paracingulate gyri function is impaired in patients with NVG and that vision is also affected. We can also hypothesize that the function of the limbic system of patients with NVG may also be affected.

The cortex adjacent to the insula is termed the operculum and is composed of three lobes. The lobes' function is important in a variety of neurological and psychiatric conditions, including sensory, motor, autonomous, and 

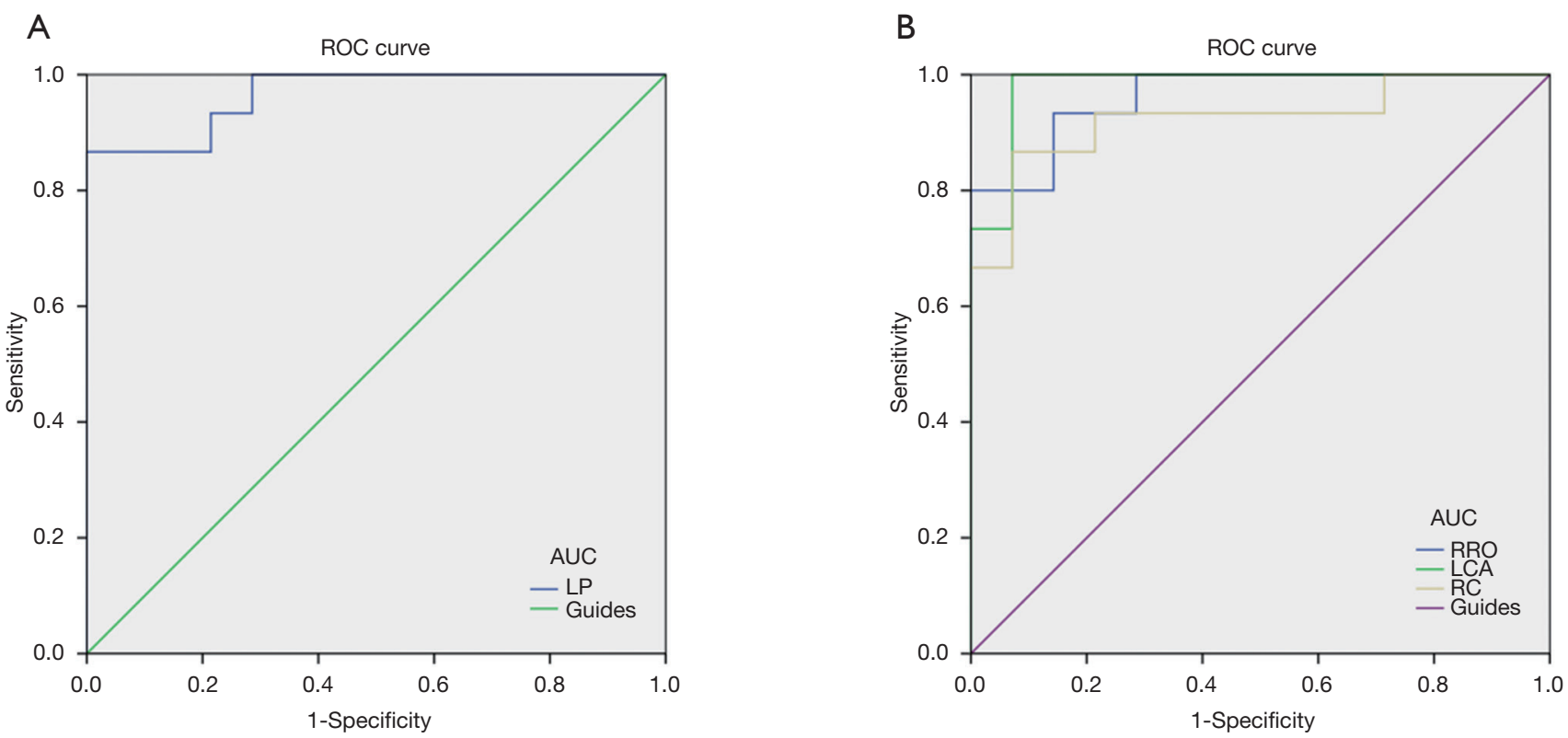

Figure 3 ROC curve based on the average fALFF value of NVG patients and HCs. It was assumed that the difference in fALFF values might be a potentially useful diagnostic marker to distinguish the NVG group from the HCs group. The greater the area under the curve (AUC), the closer the curve is to the upper left corner, and the higher the diagnostic accuracy. The AUC of the following fALFF values in different brain regions was determined: (A) the area under the ROC curve were 0.967 (P<0.001; 95\% CI: 0.911-1.000) for LP; (B) the area under the ROC curve were 0.962 (P<0.001; 95\% CI: 0.903-1.000) for RRO; LCA 0.981 ( $\mathrm{P}<0.001$; 95\% CI: 0.939-1.000); RC 0.924 $(\mathrm{P}<0.001 ; 95 \%$ CI: 0.820-1.000). fALFF, fractional amplitude of low-frequency fluctuation; NVG, neovascular glaucoma; HC, healthy control; AUC, area under the curve; ROC, receiver operating characteristic; LP, left precuneus; RRO, right Rolandic operculum; LCA, left anterior cingulate and paracingulate gyri; RC, right caudate.
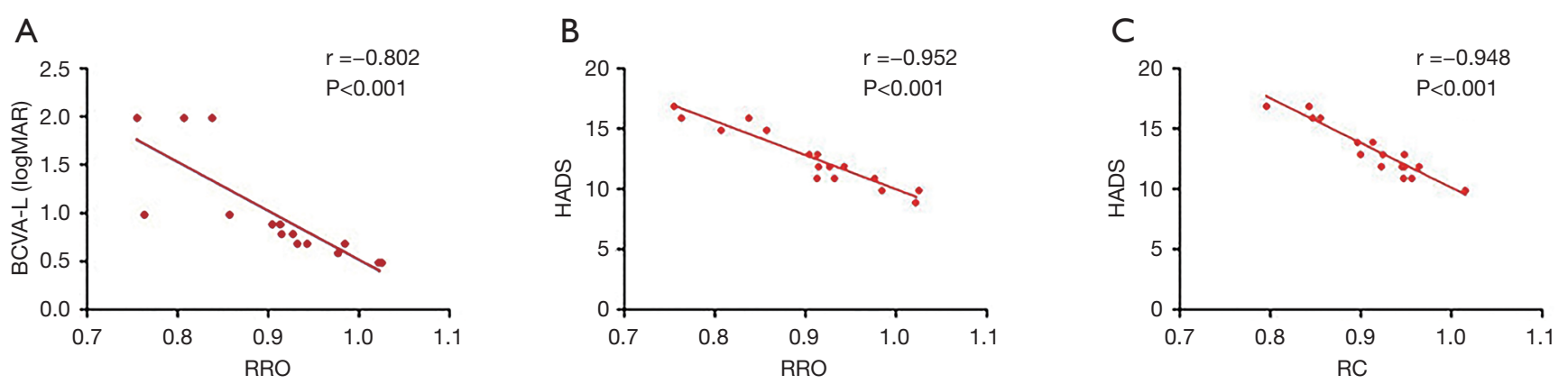

Figure 4 Correlation between relevant clinical evaluation indicators and brain regions in NVG patients. (A) Correlation between the BCVA-L and fALFF value of RRO in the NVG group. This figure shows a negative correlation between the BCVA-L and the fALFF signal value of RRO ( $\mathrm{r}=-0.802, \mathrm{P}<0.001)$. (B) Correlation between HADS and the fALFF value of RRO in the NVG group. This figure shows a negative correlation between HADS and the fALFF signal value of RRO ( $r=-0.952, \mathrm{P}<0.001)$. (C) Correlation between HADS and the fALFF value of RC in the NVG group. This figure shows a negative correlation between HADS and the fALFF signal value of RC ( $\mathrm{r}=-0.948$, $\mathrm{P}<0.001)$. BCVA-L, left best corrected visual acuity; HADS, hospital anxiety and depression scale; RRO, right Rolandic operculum; RC, right caudate; fALFF, fractional amplitude of low-frequency fluctuation; NVG, neovascular glaucoma. 
Table 4 fALFF applied in neurodegenerative diseases

\begin{tabular}{lcccc}
\hline \multirow{2}{*}{ Author } & Year & Disease & \multicolumn{2}{c}{ Brain areas } \\
\cline { 3 - 4 } & & Parkinson's disease & fALFF increased & fALFF decreased \\
\hline Tang Y, et al. (23) & 2017 & RCPL \\
Guo Z, et al. (24) & 2017 & Alzheimer's disease & RFG, LCN, RMTG & SMA \\
Sarappa C, et al. (25) & 2017 & Huntington's disease & RITG, BCL (VI, VII, IX) & BPL, SFG \\
Ma X, et al. (26) & 2016 & Amyotrophic lateral & Slow-4: RCN, LSFG, RACC; & Slow-4: RIOG, BMOG; \\
& & sclerosis & Slow-5: RMFG & Slow-5: LMOG \\
\hline
\end{tabular}

fALFF, fractional amplitude of low-frequency fluctuation; RCPL, right cerebellum posterior lobe; RFG, right fusiform gyrus; LCN, left caudate nucleus; RMTG, right middle temporal gyrus; SMA, supplementary motor area; RITG, right inferior temporal gyrus; BCL, bilaterally cerebellar lobules; BPL, bilaterally parietal lobes; SFG, superior frontal gyri; Slow-4, slow-4 band; Slow-5, slow-5 band; RCN, right caudate nucleus; LSFG, left superior frontal gyrus; RACC, right anterior cingulate cortex; RMFG, right middle frontal gyrus; RIOG, right inferior occipital gyrus; BMOG, bilateral middle occipital gyrus; LMOG, left middle occipital gyrus.

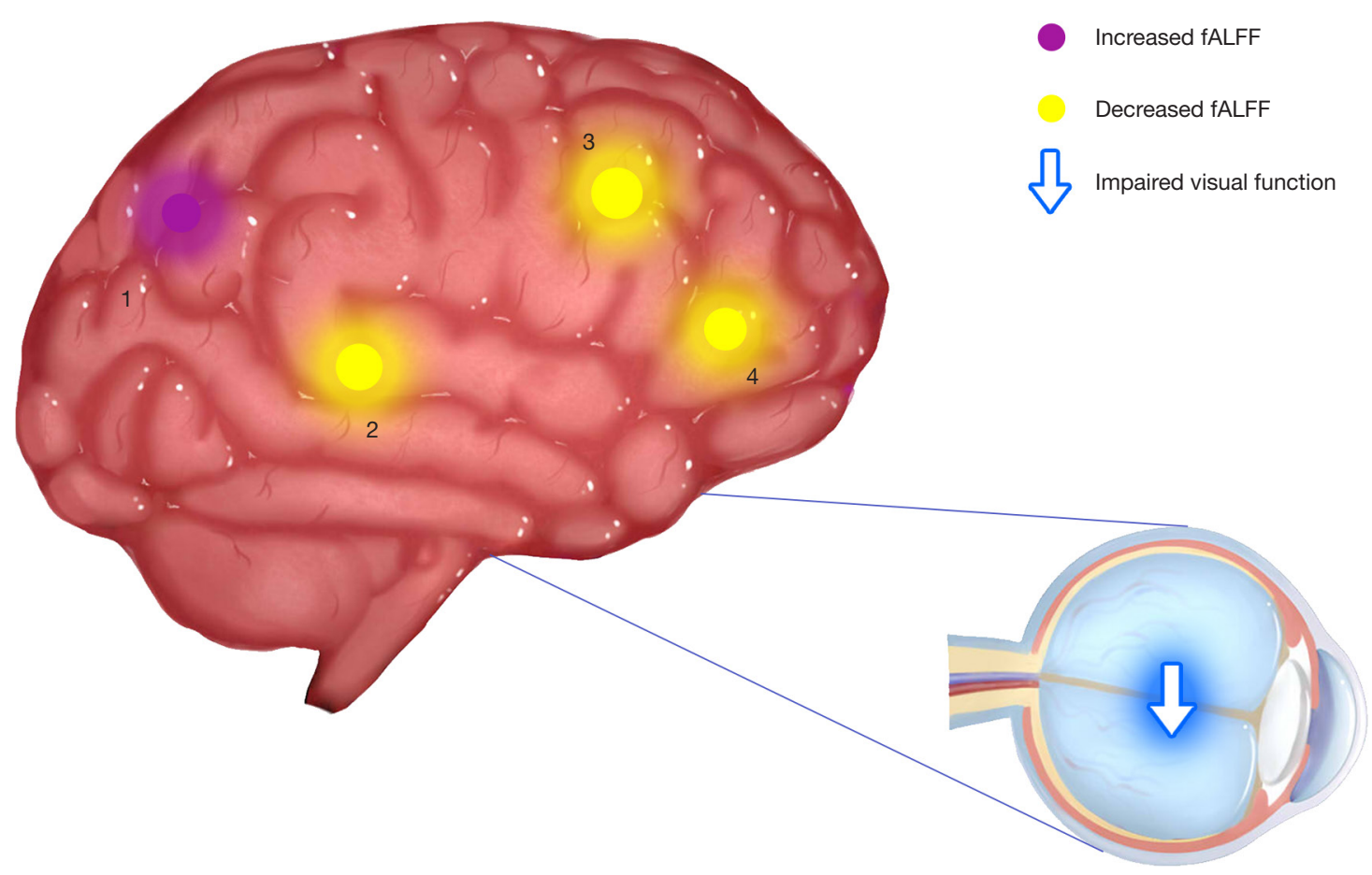

Figure 5 Specific brain regions with significant differences in internal brain activity between the NVG and HC groups. Compared with the HCs, the fALFF of NVG patients in the precuneus was increased. 1: LP ( $t=3.9329)$; in these positions, the fALFF of NVG patients was reduced to varying degrees: 2: RRO ( $t=-4.3576)$; 3 : LCA ( $t=-5.1229)$; 4: RC $(t=-4.1344)$. NVG, neovascular glaucoma; HC, healthy control; fALFF, fractional amplitude of low-frequency fluctuation; LP, left precuneus; RRO, right Rolandic operculum; LCA, left anterior cingulate and paracingulate gyri; $\mathrm{RC}$, right caudate.

cognitive processing (39). Blefari et al. (40) found that the bilateral Rolandic operculum adjacent to the island process participates in the processing and perception of sensory signals, as well as in bodily self-consciousness-related processes based on cardiac visual stimulation. Research data also confirmed the role of the Rolandic operculum and neighboring areas, including the insular, in processing sensory signals related to other conscious operations such 
as visual awareness (41). Functional breakdown of the operculum can affect the nervous system and cause mental distress. In this study, we found that the fALFF value of RRO in patients with NVG was significantly reduced compared to HCs. We hypothesize that NVG involves significant damage to the Rolandic operculum. The reduced regional activity of the Rolandic operculum may reflect impaired visual function, and the psychological mood of patients with NVG may also be affected.

The caudate is a subcortical nucleus of gray matter, divided into dorsal and ventral areas according to their connectivity and functions (42). Considering connectivity is the basis of function, Huang et al. (43) adopted a segmentation method based on functional connectivity using fMRI data in a quiescent state. When combined with the clustering method, caudate functional subregions were expected to be generated. This approach resulted in a cauda cluster with connections, including the auxiliary motor region, posterior cingulate cortex, temporoparietal junction, and hippocampus. Another cluster corresponding to the cauda abdomen's central part is functionally connected to the anterior cingulate cortex, parietal lobule, putamen, orbital frontal cortex, and primary visual cortex. Together with the putamen and pale globules, the caudate forms the basal ganglia, which can regulate cortical excitability and sensory processing (44). Our results showed that the RC's fALFF value in the NVG group was significantly decreased, along with the activity of corresponding brain regions, which may affect the function of caudate and visual conduction. This may also partly explain the higher incidence of anxiety and depression in patients with glaucoma (29.66\% and $27.76 \%$, respectively), shown by Zhang et al. (45).

\section{Conclusions}

NVG is a complex neurodegenerative disease. Our research shows that spontaneous activity is abnormal in certain areas of the brain of NVG patients, revealed the potential neural mechanism of abnormal brain activity in patients with NVG, and attempted to provide some insights for the further study of NVG. There were some limitations to this study. The sample size was small, and future studies with larger samples are warranted to confirm the present findings' accuracy. Also, the subjects were recruited from a single-center, which may result in selection bias. Finally, the inclusion and exclusion criteria for the NVG group need to be further refined. Neuroprotective drugs are gradually being used for the treatment of NVG. It is worth noting that interventions focusing on patients' psychological states with NVG should also be implemented early. We hope that our present research results can assist in the treatment and further study of NVG.

\section{Acknowledgments}

Funding: This study was supported by the National Natural Science Foundation of China (No: 81660158); Natural Science Key Project of Jiangxi Province (No: 20161ACB21017); Youth Science Foundation of Jiangxi Province (No: 20151BAB215016, 20161BAB215198); Key Research Foundation of Jiangxi Province (No: 20151 B BG7 0223 , 20181 BBG70004); Excellent Talents Development Project of Jiangxi Province (S2019RCQNB0259).

\section{Footnote}

Conflicts of Interest: All authors have completed the ICMJE uniform disclosure form (available at http://dx.doi. org/10.21037/qims-20-855). The authors have no conflicts of interest to declare.

Ethical Statement: This study was approved by the Medical Ethics Committee of the First Affiliated of Nanchang University and all subjects provided informed consent before their participation.

Open Access Statement: This is an Open Access article distributed in accordance with the Creative Commons Attribution-NonCommercial-NoDerivs 4.0 International License (CC BY-NC-ND 4.0), which permits the noncommercial replication and distribution of the article with the strict proviso that no changes or edits are made and the original work is properly cited (including links to both the formal publication through the relevant DOI and the license). See: https://creativecommons.org/licenses/by-nc-nd/4.0/.

\section{References}

1. Wong TY, Chong EW, Wong WL, Rosman M, Aung T, Loo JL, Shen S, Loon SC, Tan DT, Tai ES, Saw SM; Singapore Malay Eye Study Team. Prevalence and causes of low vision and blindness in an urban malay population: the Singapore Malay Eye Study. Arch Ophthalmol 2008;126:1091-9. 
2. Narayanaswamy A, Baskaran M, Zheng Y, et al. The prevalence and types of glaucoma in an urban Indian population: the Singapore Indian Eye Study. Invest Ophthalmol Vis Sci 2013;54:4621-7.

3. Zhao X, Wang Z, Yang X. Management of neovascular glaucoma with intravitreal ranibizumab, panretinal photocoagulation, and subsequent 5-fluorouracil augmented trabeculectomy: A case report. Medicine (Baltimore) 2017;96:e7221. Erratum in: Medicine (Baltimore). 2017 Sep 15;96(37):e8130. doi: 10.1097/ MD.0000000000008130. eCollection 2017 Sep.

4. Weiss DI, Shaffer RN, Nehrenberg TR. Neovascular gluacoma complicating carotid-cavernous fistula. Arch Ophthalmol 1963;69:304-7.

5. Smith RJH. Chandler and Grant's Glaucoma. Br J Ophthalmol 1987;71:76.

6. Havens SJ, Gulati V. Neovascular Glaucoma. Dev Ophthalmol 2016;5 5:196-204.

7. Davis BM, Crawley L, Pahlitzsch M, Javaid F, Cordeiro MF. Glaucoma: the retina and beyond. Acta Neuropathol 2016;132:807-26.

8. Ramirez AI, de Hoz R, Salobrar-Garcia E, Salazar JJ, Rojas B, Ajoy D, López-Cuenca I, Rojas P, Triviño A, Ramírez JM. The Role of Microglia in Retinal Neurodegeneration: Alzheimer's Disease, Parkinson, and Glaucoma. Front Aging Neurosci 2017;9:214.

9. Ghiso JA, Doudevski I, Ritch R, Rostagno AA. Alzheimer's disease and glaucoma: mechanistic similarities and differences. J Glaucoma 2013;22 Suppl 5:S36-8. Erratum in: J Glaucoma. 2013 Sep;22(7):597-8. Doudevski, Ivo [added]; Ritch, Robert [added]; Rostagno, Agueda A [added].

10. Danesh-Meyer HV, Levin LA. Glaucoma as a neurodegenerative disease. J Neuroophthalmol 2015;35 Suppl 1:S22-8.

11. Li T, Liu Z, Li J, Liu Z, Tang Z, Xie X, Yang D, Wang N, Tian J, Xian J. Altered amplitude of low-frequency fluctuation in primary open-angle glaucoma: a resting-state FMRI study. Invest Ophthalmol Vis Sci 2014;56:322-9.

12. Chen L, Li S, Cai F, Wu L, Gong H, Pei C, Zhou F, Zeng $\mathrm{X}$. Altered functional connectivity density in primary angle-closure glaucoma patients at resting-state. Quant Imaging Med Surg 2019;9:603-14.

13. Huang X, Zhong YL, Zeng XJ, Zhou F, Liu XH, Hu PH, Pei CG, Shao Y, Dai XJ. Disturbed spontaneous brain activity pattern in patients with primary angle-closure glaucoma using amplitude of low-frequency fluctuation: a
fMRI study. Neuropsychiatr Dis Treat 2015;11:1877-83.

14. Li HL, Chou XM, Liang Y, Pan T, Zhou Q, Pei CG, Jiang J, Li B, Shao Y. Use of rsfMRI-fALFF for the detection of changes in brain activity in patients with normal-tension glaucoma. Acta Radiol 2021;62:414-22.

15. Greicius M. Resting-state functional connectivity in neuropsychiatric disorders. Curr Opin Neurol 2008;21:424-30.

16. Liu F, Hu M, Wang S, Guo W, Zhao J, Li J, Xun G, Long Z, Zhang J, Wang Y, Zeng L, Gao Q, Wooderson SC, Chen J, Chen H. Abnormal regional spontaneous neural activity in first-episode, treatment-naive patients with late-life depression: a resting-state fMRI study. Prog Neuropsychopharmacol Biol Psychiatry 2012;39:326-31.

17. Liu F, Guo W, Fouche JP, Wang Y, Wang W, Ding J, Zeng L, Qiu C, Gong Q, Zhang W, Chen H. Multivariate classification of social anxiety disorder using whole brain functional connectivity. Brain Struct Funct 2015;220:101-15.

18. Guo WB, Liu F, Xue ZM, Xu XJ, Wu RR, Ma CQ, Wooderson SC, Tan CL, Sun XL, Chen JD, Liu ZN, Xiao CQ, Chen HF, Zhao JP. Alterations of the amplitude of low-frequency fluctuations in treatment-resistant and treatment-response depression: a resting-state fMRI study. Prog Neuropsychopharmacol Biol Psychiatry 2012;37:153-60.

19. Biswal BB, Mennes M, Zuo XN, Gohel S, Kelly C, Smith SM, Beckmann CF, Adelstein JS, Buckner RL, Colcombe S, Dogonowski AM, Ernst M, Fair D, Hampson M, Hoptman MJ, Hyde JS, Kiviniemi VJ, Kötter R, Li SJ, Lin CP, Lowe MJ, Mackay C, Madden DJ, Madsen KH, Margulies DS, Mayberg HS, McMahon K, Monk CS, Mostofsky SH, Nagel BJ, Pekar JJ, Peltier SJ, Petersen SE, Riedl V, Rombouts SA, Rypma B, Schlaggar BL, Schmidt S, Seidler RD, Siegle GJ, Sorg C, Teng GJ, Veijola J, Villringer A, Walter M, Wang L, Weng XC, WhitfieldGabrieli S, Williamson P, Windischberger C, Zang YF, Zhang HY, Castellanos FX, Milham MP. Toward discovery science of human brain function. Proc Natl Acad Sci U S A 2010;107:4734-9.

20. Bullmore E, Sporns O. Complex brain networks: graph theoretical analysis of structural and functional systems. Nat Rev Neurosci 2009;10:186-98.

21. Smitha KA, Akhil Raja K, Arun KM, Rajesh PG, Thomas B, Kapilamoorthy TR, Kesavadas C. Resting state fMRI: A review on methods in resting state connectivity analysis and resting state networks. Neuroradiol J 2017;30:305-17. 
22. Dai XJ, Liu CL, Zhou RL, Gong HH, Wu B, Gao L, Wang YX. Long-term total sleep deprivation decreases the default spontaneous activity and connectivity pattern in healthy male subjects: a resting-state fMRI study. Neuropsychiatr Dis Treat 2015;11:761-72.

23. Tang Y, Meng L, Wan CM, Liu ZH, Liao WH, Yan XX, Wang XY, Tang BS, Guo JF. Identifying the presence of Parkinson's disease using low-frequency fluctuations in BOLD signals. Neurosci Lett 2017;645:1-6.

24. Guo Z, Liu X, Li J, Wei F, Hou H, Chen X, Li X, Chen W. Fractional amplitude of low-frequency fluctuations is disrupted in Alzheimer's disease with depression. Clin Neurophysiol 2017;128:1344-9.

25. Sarappa C, Salvatore E, Filla A, Cocozza S, Russo CV, Saccà F, Brunetti A, De Michele G, Quarantelli M. Functional MRI signal fluctuations highlight altered resting brain activity in Huntington's disease. Brain Imaging Behav 2017;11:1459-69.

26. Ma X, Zhang J, Zhang Y, Chen H, Li R, Long Z, Zheng J, Wang J, Chen H. Frequency-specific alterations in the fractional amplitude of low-frequency fluctuations in amyotrophic lateral sclerosis. Neurol Sci 2016;37:1283-91.

27. Zou QH, Zhu CZ, Yang Y, Zuo XN, Long XY, Cao QJ, Wang YF, Zang YF. An improved approach to detection of amplitude of low-frequency fluctuation (ALFF) for resting-state fMRI: fractional ALFF. J Neurosci Methods 2008;172:137-41.

28. Crish SD, Sappington RM, Inman DM, Horner PJ, Calkins DJ. Distal axonopathy with structural persistence in glaucomatous neurodegeneration. Proc Natl Acad Sci U S A 2010;107:5196-201.

29. Baker CM, Burks JD, Briggs RG, Conner AK, Glenn CA, Manohar K, Milton CK, Sali G, McCoy TM, Battiste JD, O'Donoghue DL, Sughrue ME. A Connectomic Atlas of the Human Cerebrum-Chapter 8: The Posterior Cingulate Cortex, Medial Parietal Lobe, and Parieto-Occipital Sulcus. Oper Neurosurg (Hagerstown) 2018;15:S350-71.

30. Bzdok D, Heeger A, Langner R, Laird AR, Fox PT, Palomero-Gallagher N, Vogt BA, Zilles K, Eickhoff SB. Subspecialization in the human posterior medial cortex. Neuroimage 2015;106:55-71.

31. Glasser MF, Coalson TS, Robinson EC, Hacker CD, Harwell J, Yacoub E, Ugurbil K, Andersson J, Beckmann CF, Jenkinson M, Smith SM, Van Essen DC. A multimodal parcellation of human cerebral cortex. Nature 2016;536:171-8.

32. Cavanna AE, Trimble MR. The precuneus: a review of its functional anatomy and behavioural correlates. Brain 2006;129:564-83.

33. Land MF. Do we have an internal model of the outside world? Philos Trans R Soc Lond B Biol Sci 2014;369:20130045.

34. Bruner E, Iriki A. Extending mind, visuospatial integration, and the evolution of the parietal lobes in the human genus. Quat Int 2016;45:98-110.

35. Peer M, Salomon R, Goldberg I, et al. Brain system for mental orientation in space, time, and person. Proc Natl Acad Sci U S A 2015;112:11072-7.

36. Schettino A, Loeys T, Delplanque S, Pourtois G. Brain dynamics of upstream perceptual processes leading to visual object recognition: a high density ERP topographic mapping study. Neuroimage 2011;55:1227-41.

37. Shinoura N, Yamada R, Tabei Y, Shiode T, Itoi C, Saito S, Midorikawa A. The right dorsal anterior cingulate cortex may play a role in anxiety disorder and visual function. Neurol Res 2013;35:65-70.

38. Ford KA, Goltz HC, Brown MR, Everling S. Neural processes associated with antisaccade task performance investigated with event-related FMRI. J Neurophysiol 2005;94:429-40.

39. Mălîia MD, Donos C, Barborica A, Popa I, Ciurea J, Cinatti S, Mîndruţă I. Functional mapping and effective connectivity of the human operculum. Cortex 2018;109:303-21.

40. Blefari ML, Martuzzi R, Salomon R, Bello-Ruiz J, Herbelin B, Serino A, Blanke O. Bilateral Rolandic operculum processing underlying heartbeat awareness reflects changes in bodily self-consciousness. Eur J Neurosci 2017;45:1300-12.

41. Salomon R, Ronchi R, Dönz J, Bello-Ruiz J, Herbelin B, Martet R, Faivre N, Schaller K, Blanke O. The Insula Mediates Access to Awareness of Visual Stimuli Presented Synchronously to the Heartbeat. J Neurosci 2016;36:5115-27.

42. Robinson JL, Laird AR, Glahn DC, Blangero J, Sanghera MK, Pessoa L, Fox PM, Uecker A, Friehs G, Young KA, Griffin JL, Lovallo WR, Fox PT. The functional connectivity of the human caudate: an application of metaanalytic connectivity modeling with behavioral filtering. Neuroimage 2012;60:117-29.

43. Huang H, Nguyen PT, Schwab NA, Tanner JJ, Price CC, Ding M. Mapping Dorsal and Ventral Caudate in Older Adults: Method and Validation. Front Aging Neurosci 2017;9:91. 
44. Villablanca JR. Why do we have a caudate nucleus? Acta Neurobiol Exp (Wars) 2010;70:95-105.

45. Zhang D, Fan Z, Gao X, Huang W, Yang Q, Li Z, Lin M,
Xiao H, Ge J. Illness uncertainty, anxiety and depression in Chinese patients with glaucoma or cataract. Sci Rep 2018;8:11671.

Cite this article as: Zhang YQ, Peng MY, Wu SN, Yu CY, Chen SY, Tan SW, Shao Y, Zhou Q. Fractional amplitude of low-frequency fluctuation in patients with neovascular glaucoma: a resting-state functional magnetic resonance imaging study. Quant Imaging Med Surg 2021;11(5):21382150. doi: 10.21037 /qims-20-855 